\section{Intermediärrisiko-Prostatakarzinom: Hypofraktionierte RT ohne erhöhte Spättoxizität}

\begin{abstract}
Hypofraktionierte Schemata erleichtern die Radiotherapie (RT). Ob Effektivität und Toxizität vergleichbar sind mit Standardregimes, wurde in einer prospektiven Studie beim Prostatakarzinom mit intermediärem Risiko untersucht.
\end{abstract}

\begin{abstract}
ntensitätsmodulierte und 3D-konformale RT-Schemata ermöglichen eine zielgerichtete Behandlung des Prostatakarzinoms mit geringer Toxizität auf Blase und Rektum. Das erleichtert den Einsatz in hypofraktionierten Therapieprotokollen mit höheren Strahlendosen in weniger Sitzungen. Aufgrund des niedrigen $\alpha / \beta$-Werts des Tumorgewebes lässt sich auch die Gesamtstrahlendosis reduzieren.

In einer Nichtunterlegenheitsstudie sollte dieses Vorgehen beim Intermediärrisiko-Prostatakarzinom im Hinblick auf Effektivität und Spättoxizitäten überprüft werden. Einbezogen waren Patienten mit einem bezüglich GleasonScore und PSA stratifizierten interme-
\end{abstract}

diären Risiko. Randomisiert unterzogen sie sich einer konventionellen RT mit 78 Gy in 39 Fraktionen über 8 Wochen oder einer hypofraktionierten RT mit $60 \mathrm{~Gy}$ in 20 Fraktionen über 4 Wochen. Primärer Studienendpunkt war das biochemisch-klinische Rezidiv (BCF), definiert als PSA-Anstieg (Nadir $+2 \mathrm{ng} /$ $\mathrm{mL}$ ), Notwendigkeit einer Hormontherapie, klinisches Rezidiv oder karzinombedingter Tod. Als Nichtunterlegenheitsgrenze wurde eine maximale Abweichung von 7,5\% (Hazard Ratio $[\mathrm{HR}]<1,32$ ) festgelegt.

Nach median 6 Jahren hatten 109 von 608 Patienten in der Hypofraktionierungsgruppe versus 117 der $598 \mathrm{im} \mathrm{Stan-}$ dard-RT-Arm ein BCF erlitten, meist in
Form eines PSA-Anstiegs. Das BCF-freie 5-Jahres-Überleben errechnete sich mit jeweils $85 \%$ in beiden Gruppen (HR 0,96, $90 \%$-Konfindenzintervall 0,77-1,2). In der Kurztherapiegruppe ereigneten sich 10 tumorbedingte Todesfälle im Vergleich zu 12 im Standardarm. Unterschiede bei den Spättoxizitäten vom Grad $\geq 3$ im Urogenital- und Gastrointestinaltrakt gab es nicht, bei den GINebenwirkungen ließ sich ein Trend zuungunsten der Standard-RT erkennen.

Fazit: Bei Patienten mit einem Intermediärrisiko-Prostatakarzinom erwies sich ein 4-wöchiges hypofraktioniertes Bestrahlungsschema einem 8-wöchigen Standardschema beim primären Studienziel BCF als nicht unterlegen. Erhöhte Raten von Spättoxizitäten im Urogenitalund Gastrointestinaltrakt ergaben sich im median 6-jährigen Beobachtungszeitraum nicht.

Barbara Kreutzkamp

Catton $\mathrm{CN}$ et al. Randomized trial of a hypofractionated radiation regimen for the treatment of localized prostate cancer. J Clin Oncol. 2017;35(17):1884-90.

\section{Photodynamische Lokaltherapie versus Active Surveillance beim Niedrigrisiko-Prostatakarzinom}

\author{
Fokale Therapien sind eine zunehmend interessante Alternative zur aktiven \\ Beobachtung beim Niedrigrisiko-Prostatakarzinom. Für die photodynamische \\ Lokaltherapie mit Padeliporfin liegen jetzt die Ergebnisse einer großen \\ Phase-III-Vergleichsstudie vor.
}

$\mathrm{D}$ ie lokale blutgefäßgerichtete photodynamische Therapie (PDT) ist eine neue Alternative beim NiedrigrisikoProstatakarzinom. Bei diesem Eingriff werden unter transrektaler Ultraschallkontrolle eine oder mehrere dünne optische Fasern in das vorher durch MRTBildgebung festgelegte Behandlungsareal der Prostata platziert. Nach intravenöser Applikation des Photosensitizers Padeliporfin wird dieser dann lokal über die optischen Fasern durch Laserlicht (753 nm) aktiviert, und es kommt durch die phototoxische Reaktion zum Verschluss der tumornahen Gefäße.

In einer multizentrischen Phase-IIIStudie wurde die teilablative Lokalthe- rapie mit dem Standard Actice Surveillance - mit Biopsien und PSA-Wert-Bestimmung alle 12 Monate und digitaler rektaler Untersuchung alle 3 Monate verglichen. Randomisiert wurden die Patienten mit lokalisiertem Niedrigrisiko-Prostatakarzinom (Gleason-Score 3) einer von 2 Gruppen zugeordnet: Die eine Gruppe unterzog sich der PDT $(\mathrm{n}=206)$, die andere Gruppe wurde aktiv überwacht $(n=207)$. Primäre Endpunkte waren eine histologische Progression und ein Gleason-Score-Anstieg innerhalb von 24 Monaten.

Nach median 24 Monaten war die Erkrankung bei 58 (28\%) der Patienten unter der PDT und bei 120 (58\%) unter
Active Surveillance vorangeschritten (adjustierte Hazard Ratio 0,34; $\mathrm{p}<0,0001$ ). Bei $49 \%$ der Männer in der Interventionsgruppe war die Biopsie nach $24 \mathrm{Mo}$ naten negativ, verglichen mit $14 \%$ in der Beobachtungsgruppe (adjustierte Risikostratifizierung 3,67; $p<0,0001$ ). Die gezielte photodynamische Therapie wurde gut vertragen, auffällige urogenitale $\mathrm{Ne}$ benwirkungen wurden nicht registriert.

Fazit: Bei Patienten mit NiedrigrisikoProstatakarzinom bietet die gezielte photodynamische Therapie mit partieller Prostataablation eine wirksame Alternative zu Active Surveillance. Die gut verträgliche Lokaltherapie könnte bei vielen Patienten die Lücke zwischen Nichtbehandlung und radikaler Therapie zumindest über einen mittelfristigen Zeitraum füllen. Barbara Kreutzkamp

Azzouzi AR et al. Padeliporfin vascular-targeted photodynamic therapy versus active surveillance in men with low-risk prostate cancer (CLIN1001 PCM301): An open-label, phase 3, randomised controlled trial. Lancet Oncol. 2017;18(2):181-91. 